Bryn Mawr College

Scholarship, Research, and Creative Work at Bryn Mawr

College

2010

\title{
A proton spin-lattice relaxation rate study of methyl and t-butyl group reorientation in the solid state
}

Laura C. Popa

Arnold L. Rheingold

Peter A. Beckmann

Bryn Mawr College, pbeckman@brynmawr.edu

Let us know how access to this document benefits you.

Follow this and additional works at: http://repository.brynmawr.edu/physics_pubs

Part of the Physics Commons

\section{Custom Citation}

Lauren C. Pope, Arnold L. Rheingold, and Peter A. Backmann. 2010. "A proton spin-lattice relaxation rate study of methyl and t-butyl group reorientation in the solid state." Solid State Nuclear Magnetic Resonance 38.1: 31-35.

This paper is posted at Scholarship, Research, and Creative Work at Bryn Mawr College. http://repository.brynmawr.edu/physics_pubs/106

For more information, please contact repository@brynmawr.edu. 


\section{A proton spin-lattice relaxation rate study of methyl and $t$-butyl group reorientation in the solid state}

http://www.sciencedirect.com/science/article/pii/S0926204010000329 - sec4

Laura C. Popa ${ }^{\mathrm{a}, \dagger}$, Arnold L. Rheingold ${ }^{\mathrm{b}}$, Peter A. Beckmann ${ }^{\mathrm{a}, *}$

aDepartment of Physics, Bryn Mawr College, 101 North Merion Ave., Bryn Mawr, PA 19010-2899, USA.

${ }^{b}$ Department of Chemistry and Biochemistry, University of California, San Diego, 9500 Gilman Drive, La Jolla, CA 92093-0358, USA.

†Current Address: Department of Physics, Massachusetts Institute of Technology,

77 Massachusetts Ave., Cambridge, MA 02139-4307, USA.

*Corresponding author. Fax:+1610 5267469

E-mail address: pbeckman@brynmawr.edu (P. A. Beckmann)

key words: solid state NMR relaxation; X-ray diffraction; intramolecular motion; $t$-butyl group reorientation; methyl group reorientation 


\begin{abstract}
We have measured the solid state nuclear magnetic resonance (NMR) ${ }^{1} \mathrm{H}$ spin-lattice relaxation rate from 93 to $340 \mathrm{~K}$ at NMR frequencies of 8.5 and $53 \mathrm{MHz}$ in 5-t-butyl-4-hydroxy-2methylphenyl sulfide. We have also determined the molecular and crystal structure from X-ray diffraction experiments. The relaxation is caused by methyl and $t$-butyl group rotation modulating the spin-spin interactions and we relate the NMR dynamical parameters to the structure. A successful fit of the data requires that the 2-methyl groups are rotating fast (on the NMR time scale) even at the lowest temperatures employed. The rotational barrier for the two out-of-plane methyl groups in the $t$-butyl groups is $14.3 \pm 2.7 \mathrm{~kJ}$ mol ${ }^{1}$ and the rotational barrier for the $t$-butyl groups and their in-plane methyl groups is $24.0 \pm 4.6 \mathrm{~kJ}$ mol ${ }^{1}$. The uncertainties account for the uncertainties associated with the relationship between the observed NMR activation energy and a model-independent barrier, as well as the experimental uncertainties.
\end{abstract}

\title{
1. Introduction
}

Nuclear spin-lattice relaxation experiments in molecular solids like those discussed here probe intramolecular dynamics over time scales of approximately $10^{-12}$ to $10^{-5} \mathrm{~s}$; spanning $6-8$ orders of magnitude. As such, nuclear magnetic resonance (NMR) relaxation experiments can be very sensitive to subtle differences in chemical environments so long as measurements are made over a large temperature range and at more than one NMR frequency. The technique is most effective for compounds composed of smaller molecules with simpler crystal structures. NMR relaxation measurements do have the disadvantage that not many parameters can be determined from the experiments. This can mean that sometimes one cannot distinguish between more than one model for the motion and sometimes subtle differences in chemical environments can be seen qualitatively 
but not elucidated quantitatively. An improvement results when the NMR relaxation results can be correlated with molecular and crystal structure information obtained from X-ray diffraction experiments. X-ray diffraction experiments provide structural information based on the average of many "instantaneous snap shots" of atomic positions. The atom-X-ray photon interaction takes approximately $10^{-19} \mathrm{~s}$ which is instantaneous when compared with the time scales mentioned above. By combining NMR relaxation and X-ray diffraction results one can correlate dynamics with structure and provide conceptual, qualitative, and quantitative models for intramolecular motion in organic solids.

Methyl group and $t$-butyl group rotation modulates the ${ }^{1} \mathrm{H}-{ }^{1} \mathrm{H}$ spin-spin interactions in solids and causes a perturbed ${ }^{1} \mathrm{H}$ nuclear spin magnetization to relax to its equilibrium value [1]. As such, the observed NMR spin-lattice relaxation rate $R$ can be related to models of the motion. Models developed for the reorientation of "isolated" methyl groups [2] have been expanded to include the reorientation of $t$-butyl groups and their constituent methyl groups on planar aromatic molecules [3]. The molecular structure in this class of compounds (as determined by X-ray diffraction) shows the $t$-butyl groups oriented such that one methyl group is in the aromatic plane, or nearly so, and two methyl groups are out of the plane [3]. See Fig. 1. In this model, the in-plane methyl group reorients at the same rate as the $t$-butyl group, whereas the two out-of-plane methyl groups usually reorient more rapidly. This model is easily extended [4] to systems like that studied here (5-t-butyl-4-hydroxy-2-methylphenyl sulfide [the sulfide]; see Fig. 1) which have both $t$-butyl groups and "lone" methyl groups (meaning methyl groups not in $t$-butyl groups; in this case the 2methyl groups). In a recent study we tested the model with 4,5-dibromo-2,7-di-t-butyl-9,9dimethylxanthene (the xanthene) where the lone methyl groups (the 9-methyl groups) were found to have rotational barriers similar to the rotational barriers of the out-of-plane methyl groups in the 
$t$-butyl groups [4]. It was concluded that the rotation of the lone methyl groups was clearly discernable in the high-temperature, thermally assisted hopping regime studied, even though the relaxation rate for this lone methyl group motion occurred in the same temperature region as the relaxation rate resulting from the motion of the out-of-plane methyl groups in the $t$-butyl groups, as could be predicted by the structure of the xanthene molecule. Here, we extend this project in two directions. First, whereas the two carbon rings in the xanthene and most other systems studied to date have been coplanar, in the sulfide the backbone structure (i.e., excluding the methyl and $t$-butyl groups) is much more complex (Fig. 1). Second, on inspecting the molecular and crystal structure in the sulfide (Fig. 2), the 2-methyl groups are seen to be in a less restricted region of space and, indeed, we can determine unequivocally that we cannot "see" spin-lattice relaxation from their reorientation because they are rotating fast on the NMR time scale even at our lowest temperatures.

\section{The Experiments}

The sample, 5-t-butyl-4-hydroxy-2-methylphenyl sulfide, was purchased from Acros and carefully recrystallized. The melting point of the final product was 161-162 C.

The X-ray diffraction experiments were performed at the University of California, San Diego. A colorless block $0.50 \times 0.25 \times 0.15 \mathrm{~mm}$ was mounted on a Cryoloop with Paratone-N oil. Data were collected in a nitrogen gas stream at 100(2) K using phi and omega scans. Crystal-to-detector distance was $60 \mathrm{~mm}$ and exposure time was 10 seconds per frame using a scan width of $0.5^{\circ}$. Data collection was $100 \%$ complete to $25.00^{\circ}$ in theta using MoK $\alpha$. A total of 17623 reflections were collected covering the indices, $-22 \leq \mathrm{h} \leq 27,-9 \leq \mathrm{k} \leq 7,-35 \leq 1 \leq 29$. 4663 reflections were found to be symmetry independent, with $R$ int $=0.0482$. Indexing and unit cell refinement indicated a face-centered monoclinic lattice. The space group was found to be $\mathrm{C} 2 / \mathrm{c}$. The data were integrated 
using the Bruker APEXII software program and scaled using the SADABS software program. Solution by direct methods (SHELXS) and all non-hydrogen atoms were refined anisotropically by full-matrix, least-squares on F2 (SHELXL-97). All hydrogen atoms except H1a and H2a (those in the hydroxy groups) were constrained relative to their parent atom using the appropriate HFIX command in SHELXL-97. Hydrogen atoms H1a and H2a were found from a Fourier difference map and were allowed to refine. The molecular structure is shown in Fig. 1 and the crystal structure is shown in Fig. 2.

The ${ }^{1} \mathrm{H}$ spin-lattice relaxation rate $R$ measurements were performed at Bryn Mawr College from 93 to $340 \mathrm{~K}$ at NMR frequencies of $/ 2 \quad=8.5$ and $53 \mathrm{MHz} . R$ values were measured using an inversion-recovery pulse sequence. Further details of the measurement process and temperature control and measurement are provided elsewhere [4]. There were no thermal history effects. The spin-spin relaxation rate, characterized by the free induction decay, was approximately constant at $(10 \mathrm{~s})^{-1}$ which is several orders of magnitude greater than the largest $R$ values, indicating rapid spin-diffusion. As a consequence, all protons, involved with the motion or not, relax with the same rate. The relaxation was exponential at all temperatures, thus resulting in a uniquely defined relaxation rate $R$. $\operatorname{Ln} R$ versus inverse temperature $T^{1}$ is shown in Fig. 3 .

\section{The Spin-Lattice Relaxation Model and the NMR Fitting Parameters}

The X-ray diffraction data show that all molecules are symmetry equivalent but that the two halves of the molecule are in different environments; that is, the two $t$-butyl groups and the two 2methyl groups in the molecule are inequivalent. See Fig. 2(b) where the two different ends of the molecule are labeled 1 and 2. An inspection of the geometry, including intermolecular distances, suggests that their are no unusually close intermolecular hydrogen-hydrogen distances for either $t$ - 
butyl group. This means that the dynamical properties of the two $t$-butyl groups are not likely to be very different, as born out by fitting the relaxation rate data. $\operatorname{Ln} R$ versus $T^{1}$ in Fig. 3 shows two maxima in $R$. Using the $8.5 \mathrm{MHz}$ data for comparison with other works, these two maxima are $R=$ $68 \mathrm{~s}^{1}$ at $140 \mathrm{~K}\left(10^{3} T^{1}=7.14 \mathrm{~K}^{1}\right)$ and $R=100 \mathrm{~s}^{1}$ at $210 \mathrm{~K}\left(10^{3} T^{1}=4.8 \mathrm{~K}^{1}\right)$. The higher temperature maximum in $R$ is greater than the lower temperature maximum in $R$ and that the temperature difference between the two $R$ maxima is $70 \mathrm{~K}$. The two $R$ maxima are associated with two sets of coupled motions as discussed below. These data can be compared with similar data for other organic solids with $t$-butyl groups in references 3-6. In Fig, 4 of [3] and Fig. 3 of [4], the two maxima in $R$ have smaller separations in temperature ( $30 \mathrm{~K}$ and $50 \mathrm{~K}$, respectively) than the data reported here. In Fig. 4 of [5] and Fig. 2 of [6], the separation is greater ( $230 \mathrm{~K}$ and $90 \mathrm{~K}$ respectively) than that reported here. But the current $\ln R$ versus $T^{1}$ in Fig. 3 has the interesting feature, discussed further below, that in the temperature region studied we cannot "see" relaxation resulting from the reorientation of the two 2-methyl groups. The previous studies either had no additional "lone" methyl groups $[3,5,6]$ or the relaxation rate from the rotation of the lone methyl groups was observed in the temperature range studied [4].

We begin with the (incorrect) assumption that the two $t$-butyl groups are equivalent and the two lone methyl groups are equivalent and then add as few additional adjustable parameters as needed to rectify the incorrect assumption and make the model consistent with the data. The relaxation rate $R$ is given by [4] a sum of several terms of the form $R={ }_{i} K_{i}\left[J\left(,{ }_{i}\right)+\right.$ $\left.4 J\left(2,{ }_{i}\right)\right]$ where the ${ }_{i}$ characterize the various correlation times (mean times between instantaneous $2 / 3$ hops) and the $K_{i}$ are the accompanying time-independent constants that depend only on the geometric properties and physical constants. The $K_{i}$ are proportional to $r_{i}^{-6}$ for proton-proton separations $r_{i}$. In turn, the correlation times ${ }_{i}$ are characterized, via an Arrhenius 
relationship, by a preexponential factor $\omega_{\infty i}$ and an NMR activation energy $E_{i}$, [ ${ }_{i}={ }_{\infty} i$ $\left.\exp \left(E_{i} / k T\right)\right]$. The $E_{i}$ can then be related to the height of the rotational barriers $V_{i}$ as discussed in the Summary and Discussion section. The NMR activation energies $E_{i}$ tend to be determined with reasonable accuracy by the experiment $( \pm 10 \%)$. They should fall within well-defined norms for the kinds of motions being studied here $[3,5,7,8]$. Once the $E_{i}$ are determined, a somewhat overly simplistic, but nevertheless helpful model $[3,9,10]$ predicts the preexponential factors $\infty_{\infty} i$ to be $\tilde{}_{\infty i}=(2 / 3)\left(2 I / E_{i}\right)^{1 / 2}$ for moment of inertia $I$, and the fitted values should agree with these predicted values to within an order of magnitude or so. The fitted values of the $\infty_{\infty i}$ are then given in terms of the ratios of these values to the predicted values ${ }_{\infty}$.

Three of the terms in $R={ }_{i} K_{i}\left[J\left(,_{i}\right)+4 J\left(2,{ }_{i}\right)\right]$ correspond to (1) lone 2-methyl group rotation [characterized by the correlation time ${ }_{m}$ (i.e., $\left.i=m\right)$ ], (2) out-of-plane $t$-butyl group methyl group rotation (characterized by ${ }_{c}$ ), and (3) in-plane $t$-butyl group methyl group rotation and $t$-butyl group rotation (both of which are characterized by the same correlation time ${ }_{b}$ ). Two additional terms in this sum are (4) the superposition of out-of-plane $t$-butyl methyl group rotation and $t$-butyl group rotation (characterized by ${ }_{b c}^{1}=b^{1}+{ }_{c}^{1}$ ) and (5) the superposition of inplane $t$-butyl group methyl group rotation and $t$-butyl group rotation (characterized by $\quad b b=b^{1}+$ $\left.b^{1}=2_{b}{ }^{1}\right)$. These latter two terms do not introduce additional correlation times or adjustable parameters. Knowing the molecular and crystal structure, the five values of $K_{i}$ corresponding to the five terms above can be predicted with reasonable assumptions. The three independent theoretical $K_{i}$ values, labeled $\tilde{K}_{i},(i=b, c, m)$ have approximately $\pm 10 \%$ uncertainties [4] and the three independent fitting parameters are $K_{b} / \tilde{K}_{b}, K_{c} / \tilde{K}_{c}$, and $K_{m} / \tilde{K}_{m}$. The other two values of $K_{i}$ satisfy $K_{b b} / \tilde{K}_{b b}=K_{b} / \tilde{K}_{b}$ and $K_{b c} / \tilde{K}_{b c}=K_{c} / \tilde{K}_{c}$. The $\pm 10 \%$ uncertainties in the $\tilde{K}_{i}$ have their 
origin in the approximately $\pm 1-2 \%$ uncertainties in interatomic distances and the fact that the $\tilde{K}_{i}$ are proportional to $r^{6}$.

For motions like those studied here, the spectral density should characterize random uncorrelated hopping ("reorientation") since there is no experimental evidence that correlated motions play any role (the relaxation is strictly exponential) [11] nor is there any structural evidence that there would be any dynamical bottlenecks [12]. Thus, the spectral density for each term in $R={ }_{i} K_{i}\left[J\left(,{ }_{i}\right)+4 J\left(2,{ }_{i}\right)\right]$ should be the Debye spectral density $J\left(,_{i}\right)=2_{i} /(1$ $+2{ }_{i}^{2}$ ). However, if we assume that the two $t$-butyl groups are equivalent, this form of the spectral density will not fit the data for any reasonable set of values for the $K_{i}$, the $E_{i}$, and the $\infty i$. Doubling the number of parameters (because there are two inequivalent $t$-butyl groups) provides many possible equally successful fits of the relaxation rate data corresponding to a wide range of values in the adjustable parameters. This says that this is an unnecessary number of adjustable parameters. As such we model the inequivalent ends of the molecule [Fig. 2(b)] by introducing a Davidson-Cole spectral density $J(, D C i)=,(2 /)[\arctan (\quad D C i)] /\left[1+{ }^{2}{ }_{D C i}^{2}\right]^{/ 2}[13,14,15]$ which reduces to a Debye spectral density $J\left(,{ }_{i}\right)=2 \quad{ }_{i} /\left(\begin{array}{lll}2 & 2 & 2\end{array}\right)$ for $=1$ (in which case ${ }_{D C}{ }_{i}$ ). This introduces only one additional adjustable parameter $0<\leq 1$ that characterizes, in a phenomenological manner, the distribution of NMR correlation times ${ }_{i}$ (and therefore the distribution of barriers). The distribution is highly asymmetric with an upper cutoff for the various correlation times (corresponding to a logarithmic singularity in the distribution function) and a tail to smaller values $[14,15]$. The subscript DC on parameters [i.e., $\left.\quad D_{C i}=\infty D C i \exp \left(E_{D C i} / k T\right)\right]$ reminds us that these parameters characterize an upper-limit to this distribution. This is discussed further in the Summary and Discussion section. 
The set of parameters used to interpret the relaxation rate data, then, is the three values $(i=b$, $c, m)$ of $E_{D C i} K_{i} / \tilde{K}_{i}$, and ${ }_{\infty D C i}{ }^{\sim}{ }_{\infty D C i}$ and the distribution parameter . This seems like many adjustable parameters but they all have quite severe constraints and none depend on temperature or NMR frequency. As a result several interesting conclusions are possible. If two NMR activation energies are involved in the model and they are sufficiently different so as to significantly separate the two maxima in $R$ versus $T^{1}$, as is the case here (Fig. 3), the larger of the two NMR uppercutoff activation energies is fixed by the frequency independent linear high-temperature $\ln R$ versus $T^{1}$ regime. In the present case, this results in the upper-cutoff for the activation energy for the reorientation of the $t$-butyl groups and their in-plane methyl groups having the value $E_{D C b}=21.6$ $\pm 2.2 \mathrm{~kJ} \mathrm{~mol}{ }^{1}$. The Davidson-Cole spectral density predicts that the frequency dependence of the linear low-temperature $\ln R$ versus $T^{1}$ regime is $R\left({ }_{1}\right) / R\left({ }_{2}\right)=\left({ }_{2} /{ }_{1}\right)^{1+}$ and this fixes the distribution parameter at $=0.65 \pm 0.03$. The fit that goes through the data in Fig. 3 adds the parameters $K_{b} / \tilde{K}_{b}=1.2 \pm 0.2$ and ${ }_{\infty D C b} /{ }_{\infty D C b}=0.17$ for the reorientation of the $t$-butyl groups and their in-plane methyl groups and $E_{D C c}=12.9 \pm 1.3 \mathrm{~kJ}$ mol ${ }^{1}, K_{c} / \tilde{K}_{c}=1.0 \pm 0.2$, and $\infty D C c^{\prime}{ }_{\infty D C c}=1.5$ for the reorientation of the out-of-plane methyl groups. The uncertainties in the $K_{i} / \tilde{K}_{i}$ have a $\pm 10 \%$ experimental contribution from $K_{i}$ and a $\pm 10 \%$ theoretical contribution from $\tilde{K}_{i}$. The uncertainties in all values of ${ }_{\infty D C i} /{ }_{\infty D C i}$ are about a factor of 3 if the activation energies are permitted to take on their upper and lower limits. (In any event, the theoretical parameters $\tilde{}_{\infty D C i}$ with which the fitted values are being compared are, themselves, order-ofmagnitude estimates $[3,9,10]$. On the other hand, if the ${ }_{\infty D C i} /{ }_{\infty D C i}$ were several orders of magnitude from unity, it would indicate an inappropriate model.)

Further, the 2-methyl group is reorienting sufficiently rapidly that its contribution to the observed relaxation rate is not observed. That is, $E_{D C m}$ is less than about $3.5 \mathrm{~kJ}$ mol ${ }^{1}$ which 
makes $D_{D C m}=\infty D C m \exp \left(E_{D C m} / k T\right)<\left(10^{-3}\right) \quad 1_{\text {at }}$ the lowest temperatures used. If the reorientation of these lone methyl groups is characterized by the same parameters as the out-ofplane methyl groups in the $t$-butyl groups for comparison sake (that is ${ }_{\infty} D C m=\infty D C c$ and $E_{D C m}$ $=E_{D C c}$ ), the total relaxation would be the upper line in Fig. 3. No reasonable adjustment of the parameters results in any part of the observed relaxation being due to the reorientation of the 2methyl groups.

\section{Summary and Discussion}

The molecular structure of 5-t-butyl-4-hydroxy-2-methylphenyl sulfide (the sulfide) is shown in Fig. 1 and the crystal structure is shown in Fig. 2. The ${ }^{1} \mathrm{H}$ spin-lattice relaxation rate as a function of temperature at two NMR frequencies is shown in Fig. 3. It is interesting to compare the data in Fig. 3 with the similar figure for 4,5-dibromo-2,7-di-t-butyl-9,9-dimethylxanthene (the xanthene) [4]. Both molecules have $t$-butyl groups and methyl groups not in $t$-butyl groups. Both show a low-temperature maximum in $R$ (at $130 \mathrm{~K}$ in the xanthene and at $140 \mathrm{~K}$ in the sulfide at $8.5 \mathrm{MHz}$ ) resulting from the rotation of the out-of-plane methyl groups in the $t$-butyl groups and the superposition of this motion and the rotation of the $t$-butyl groups. Both show a high-temperature maximum in $R$ (at $180 \mathrm{~K}$ in the xanthene and at $210 \mathrm{~K}$ in the sulfide at $8.5 \mathrm{MHz}$ ) resulting from the rotation of the $t$-butyl groups and the in-plane methyl groups and from the superimposed rotation of these two rotations. The individual contributions to $R$ are outlined in detail in Fig. 6 of [8]. In the sulfide, $R$ from the lone methyl groups is not observed because they are reorienting rapidly and their contribution to $R$ is zero (because $\omega \tau<<1$ at all temperatures for this motion). As a consequence, in the sulfide studied here, the maximum value of $R$ at the higher temperature is greater than the maximum value of $R$ at the lower temperature. In the xanthene [4], where the lone 
methyl group reorients with a correlation time that is approximately the same as the out-of-plane methyl groups in the $t$-butyl groups (as predicted by the molecular structure), the values of the maxima in $R$ are the other way around, as indicated by the upper line in Fig. 3 (and Fig. 3 of [4]). There is no question that the models can distinguish between these two cases. Preliminary field cycling relaxation experiments from the Horsewill Group in Nottingham University have confirmed that the 2-methyl groups in the sulfide undergo tunneling motion and that the two methyl groups are inequivalent, as expected [16].

The upper limit NMR activation energy for the reorientation of the out-of-plane methyl groups in the $t$-butyl groups in the sulfide (the low-temperature maximum in $R$ in Fig. 1) is $E_{D C c}=12.9 \pm$ $1.3 \mathrm{~kJ} \mathrm{~mol}{ }^{1}$, which is in the range expected for methyl groups in a $t$-butyl group where the intra-tbutyl group interactions are dominant $[3,5-7,17]$. Indeed, this activation energy is approximately the same as that for the methyl groups in an isopropyl group (which are out of the plane of an aromatic ring) $[18,19]$ or the methyl group in an ethyl group (which is also out of the plane of an aromatic ring) $[15,17,19]$.

The relationship between an NMR activation energy and a technique-independent absolute barrier height (which is the sought-after parameter) is very complicated as discussed by Kowaleski and Liljefors [20] and by Edholm and Blomberg [21]. These authors perform extensive numerical calculations for observed NMR activation energies in the range observed in this work. Their investigations can be summarized by saying that NMR activation energies can be, approximately, between zero and $20 \%$ smaller than the heights of the rotational barriers for methyl groups with activation energies in the range reported here. It must be appreciated that NMR activation energies are not absolute model-independent and technique-independent barriers. That having been said, these barriers for methyl group rotation are low compared with many other dynamical processes 
and relaxation NMR is one of the few techniques that allow methyl group rotation to be investigated in detail. As such, we incorporate this 0-20\% range into the uncertainties inherent in the modeling and report the barrier height for the rotation of the out-of-plane methyl groups in the $t$-butyl groups as $V_{D C c}=14.3 \pm 2.7 \mathrm{~kJ} \mathrm{~mol}^{1}$.

The upper-limit NMR activation energy for the $t$-butyl groups and their constituent in-plane methyl groups is $E_{D C b}=21.6 \pm 2.2 \mathrm{~kJ}$ mol ${ }^{1}$. This can be compared with similar values for a $t$ butyl group on an aromatic ring adjacent to a hydroxy group [5, 6, 8, 22]. The relationship between $t$-butyl group rotational barriers and NMR activation energies is more difficult to determine than for simple methyl group rotation but one can still say that the barrier will likely be at least somewhat larger than the activation energy because the rotational ground state will be above the bottom of the barrier. Using the same "conversion" as used for methyl groups as a guide, the upper limit for the barrier for the reorientation of the $t$-butyl groups and their constituent in-plane methyl groups will be $V_{D C b}=24.0 \pm 4.6 \mathrm{~kJ}$ mol ${ }^{1}$. As suggested by the structure determined by X-ray diffraction, the $t$-butyl groups and their constituent methyl groups on either end of the molecule [Fig (2b)] will have slightly different values of $V_{D C b}$ and $V_{D C c}$. This difference is being modeled by a distribution of barriers because that results in many fewer adjustable parameters. This does point out a limitation of relaxation rate NMR. We observe this difference in the two sets of barriers but a relaxation rate experiment does not provide enough information to fully characterize it. On the other hand, such observations, though qualitative in nature, can lead to interesting follow-up discoveries, such as the X-ray investigation into the complex crystal structure of the E polymorph of 2,6-di-t-butylnaphthalene that followed from questions raised in an NMR relaxation study.[24].

Finally, there is the matter that the quoted values of the NMR activation energies represent an upper-limit and that if the Davidson-Cole distribution of correlation times or NMR activation 
energies is interpreted strictly from the mathematical model, some methyl and $t$-butyl groups have lower NMR activation energies than the upper-limit cutoff value. However, in this case we are using this model in a phenomenological manner to mimic differences in both intramolecular and intermolecular interactions at the two ends of the molecule. That having been said, we can not rule out that the differences in the interactions at the two ends of the molecule are quantitatively inconsequential and the barriers for methyl and $t$-butyl rotation at the two ends of the molecule are essentially the same. In this case there may truly be a distribution of correlation times and, as a consequence, NMR activation energies. It is not possible to distinguish between these two cases for this compound; it is too complicated. We feel confident that the relatively large uncertainties in the reported values of the barriers cover all possible modeling choices. However, trying to understand the physical origins of the Davidson-Cole distribution of correlation times, using simpler molecules, is an ongoing project in our research group. It is possible that rotors in molecules near the surface of a crystallite or near imperfections in a crystallite might have lower rotational barriers than those in the "perfect" bulk crystalline environment where the barrier is characterized by the upper-limit cutoff value. Part of this ongoing research is to relate the value of the "distribution parameter" $\varepsilon$, which is the only additional parameter in going from a Debye spectral density to a Davidson-Cole spectral density, to the sizes of the crystallites used in the NMR relaxation experiments using scanning electron microscopy (SEM). If a significant number of crystallites have a smallest dimension below $1 \mu \mathrm{m}$, then a significant fraction of rotors will reside on or near a surface.

\section{Acknowledgements}


The authors thank Professor Frank B. Mallory (Bryn Mawr College) and Dr. Clelia W. Mallory (Bryn Mawr College and the University of Pennsylvania) for recrystallizing the commercial sample of 5-t-butyl-4-hydroxy-2-methylphenyl sulfide and Professor Frank B. Mallory for helpful discussions. We also thank Professor Anthony Horsewill of Nottingham University for verifying that that there are indeed tunneling methyl groups at very low temperatures.

\section{Supplementary Information}

CCDC 766580 contains the supplementary crystallographic data for 5-t-butyl-4-hydroxy-2methylphenyl sulfide. These data can be obtained free of charge from The Cambridge Crystallographic Data Centre via www.ccdc.cam.ac.uk/data_request/cif.

\section{References}

[1] C. P. Slichter, Principles of Magnetic Resonance, 3rd ed. (Springer-Verlag, Berlin, 1990).

[2] K. G. Conn, P. A. Beckmann, C. W. Mallory, F. B. Mallory, J. Chem. Phys. 87 (1987) 20-27.

[3] P. A. Beckmann, C. A. Buser, K. Gullifer, F. B. Mallory, C. W. Mallory, G. M. Rossi, A. L. Rheingold, J. Chem. Phys. 118 (2003) 11129-11138.

[4] P. A. Beckmann, W. G. Dougherty Jr., W. S. Kassel, Sol. State Nuc. Mag. Resonan. 36 (2009) $86-91$.

[5] P. A. Beckmann, C. Paty, E. Allocco, M. Herd, K. Kuranz, A. L. Rheingold, J. Chem. Phys. 120 (2004) 5309-5314.

[6] P. A. Beckmann, H. A. Al-Hallaq, A. M. Fry, A. M. Plofker, B. A. Roe, J. A. Weiss, J. Phys. Chem. 100 (1994) 752-753.

[7] A. L. Rheingold, A. G. DiPasquale, P. A. Beckmann, Chem. Phys. 345 (2008) 116-118. 
[8] P. A. Beckmann, A. M. Cheung, E. E. Fisch, F. A. Fusco, R. E. Herzog, N. Narasimhan, J. Chem. Phys. 84 (1986) 1959-1968.

[9] N. L. Owen, in Internal Rotation in Molecules, edited by W. J. Orville-Thomas (Wiley, New York, 1974), p. 157.

[10] S. Clough, A. Heidemann, J. Phys. C 13 (1980) 3585-3589.

[11] R. L. Hilt, P. S. Hubbard, Phys. Rev. 134 (1964) 392-398.

[12] R. G. Palmer, D. L. Stein, E. Abrahams, P. W. Anderson, Phys. Rev. Lett. 53, (1984) 958-961.

[13] D. W. Davidson, R. H. Cole, J. Chem. Phys. 19 (1951) 1484-1490.

[14] P. A. Beckmann, Phys. Rep. 171 (1988) 85-128.

[15] P. A. Beckmann, L. Happersett, A. V. Herzog, W. M. Tong, J. Chem. Phys. 95 (1991) 828-835.

[16] A. Horsewill, Department of Physics, University of Nottingham, private communication.

[17] P. A. Beckmann, K. S. Burbank, M. M. W. Lau, J. N. Ree, T. L Weber, Chem. Phys. 290 (2003) 241-250.

[18] X. Wang, A. L. Rheingold, A. G. DiPasquale, F. B. Mallory, C. W. Mallory, P. A. Beckmann, J. Chem. Phys. 128 (2008) 124502 1-3.

[19] P. A. Beckmann, C. A. Buser, C. W. Mallory, F. B Mallory, J. Mosher, Solid State Nuc. Mag. Resonan. 12 (1998) 251-256.

[20] J. Kowaleski, T. Liljefors, Chem. Phys. Lett. 64 (1979) 170-174.

[21] O. Edholm, C. Blomberg, Chem. Phys. 56 (1981) 9-14.

[22] P. A. Beckmann, F. A. Fusco, A. E. O'Neill, J. Mag. Resonan. 59 (1984) 63-70.

[23] A. L. Rheingold, J. S. Figueroa, C. Dybowski, and P. A. Beckmann, Chem. Comm. 651- 
652 (2000). [note to editor: year is volume.] 


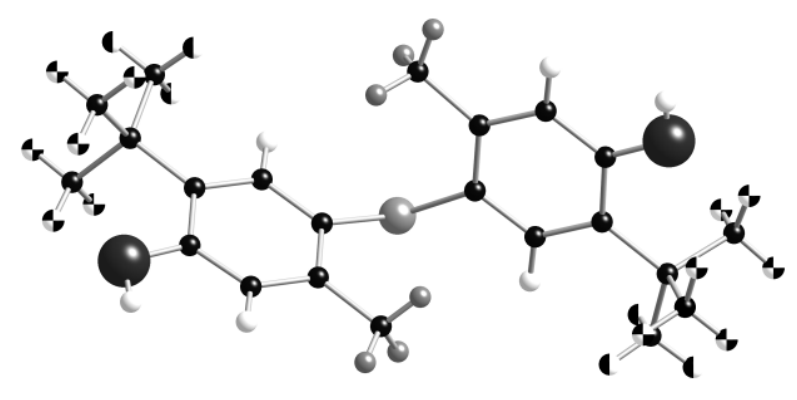

(a)

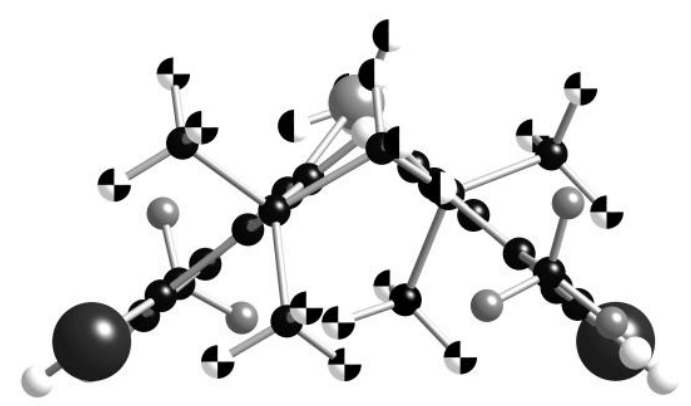

(b)

Figure 1. The molecule 5-t-butyl-4-hydroxy-2-methylphenyl sulfide with the structure it has in the crystalline state. The two large black spheres are oxygen atoms, small black spheres are carbon atoms, the large grey sphere in the center is the sulfur atom, the six small grey spheres are the two 2-methyl group hydrogen atoms, the six small white spheres are ring and hydroxy hydrogen atoms, the hydrogen atoms in the out-of-plane methyl groups in the $t$-butyl groups are indicated with beach ball markings, and the in-plane methyl groups in the $t$-butyl groups are indicated with two-tone markings. (a) An orientation that makes all atoms visible. (b) An orientation in the planes of both rings that shows the in-plane and out-of-plane $t$-butyl methyl groups more clearly. This also shows interesting distortions in the central region of the molecule. 


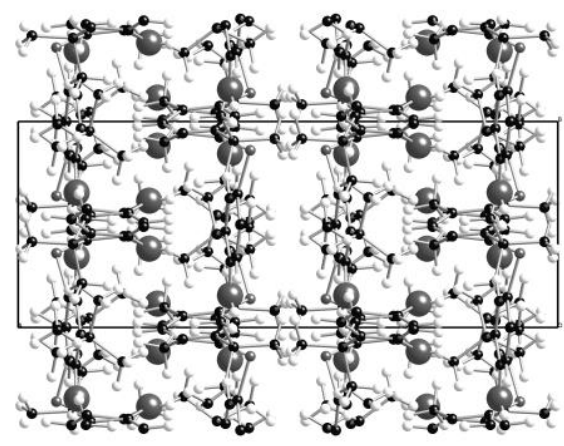

(a)

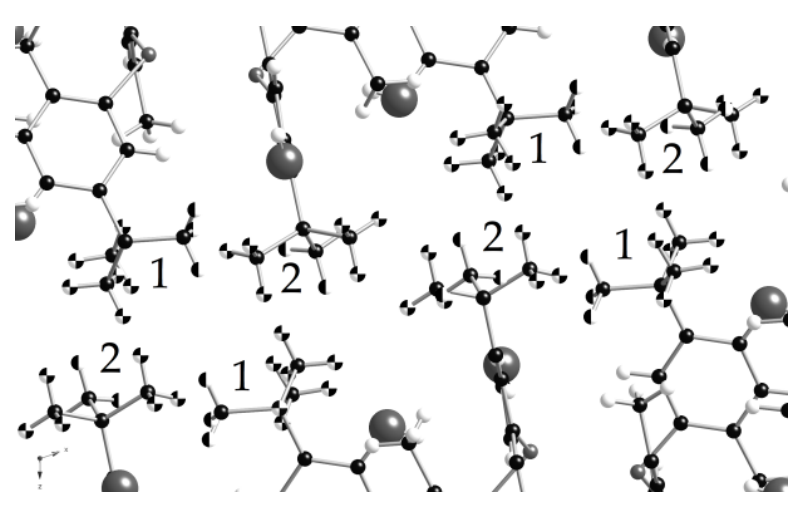

(b)

Figure 2. The crystal structure of 5-t-butyl-4-hydroxy-2-methylphenyl sulfide. (a) The 001 plane showing one unit cell in the $x$-direction (horizontal) and two unit cells in the $y$-direction (vertical). The unit cell is indicated. (b) A small part of the unit cell in the 010 direction showing the two types of $t$-butyl groups (or equivalently, the two different ends of the molecules), labeled 1 and 2. The in- and out-of-plane methyl group hydrogen atoms are indicated as explained in Fig. 1. 


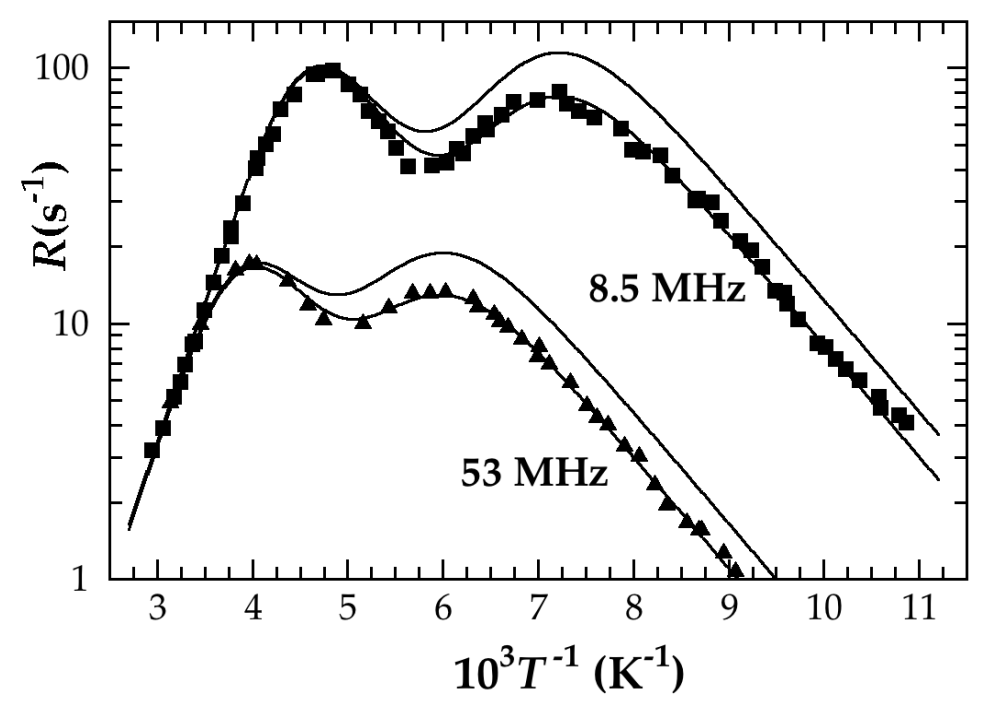

Fig. 3. The ${ }^{1} \mathrm{H}$ spin-lattice relaxation rate $R$ versus inverse temperature $T$ in polycrystalline 5-tbutyl-4-hydroxy-2-methylphenyl sulfide at $\quad / 2=8.5$ and $53 \mathrm{MHz}$ as shown. The fit going through the data assumes that the 2-methyl groups are reorienting rapidly at all temperatures shown. The higher line assumes these lone methyl groups are characterized by the same parameters that characterize the out-of-plane methyl groups in the $t$-butyl groups. 